\title{
Mythes sociotechniques et développement
}

Introduction au numéro spécial

Jean-Philippe Venot et Gert Jan Veldwisch

Traducteur : Fadhila Le Meur

\section{OpenEdition}

\section{Journals}

Édition électronique

URL : http://journals.openedition.org/anthropodev/585

DOI : 10.4000/anthropodev.585

ISSN : 2553-1719

\section{Éditeur}

Presses universitaires de Louvain

\section{Édition imprimée}

Date de publication : 1 décembre 2017

Pagination : $27-48$

ISBN : 979-10-93476-05-6

ISSN : 2276-2019

\section{Référence électronique}

Jean-Philippe Venot et Gert Jan Veldwisch, « Mythes sociotechniques et développement », Anthropologie \& développement [En ligne], 46-47 | 2017, mis en ligne le 01 juin 2018, consulté le 10 décembre 2020. URL : http://journals.openedition.org/anthropodev/585 ; DOI : https://doi.org/ 10.4000/anthropodev.585

La revue Anthropologie \& développement est mise à disposition selon les termes de la Licence Creative Commons Attribution 4.0 International. 


\title{
Mythes sociotechniques et développement
}

\author{
Introduction au numéro spécial
}

Jean-Philippe Venot, Gert Jan Veldwisch

\section{Introduction}

Au cours de ces dernières années nous avons, l'un comme l'autre, croisé des promoteurs féroces de "technologies pour le développement " telles que l'irrigation au goutte-à-goutte, l'agriculture de conservation, le système de riziculture intensive (SRI) ou, pour les plus connues, le jatropha ou les semences de coton transgénique Bt. Nous avons été frappés par le zèle avec lequel elles étaient promues et défendues (ou parfois attaquées). À y regarder de plus près, il ne s'agissait pas simplement de technologies pour le développement mais plutôt de "dispositifs sociotechniques " qui partageaient de nombreuses ressemblances: leurs définitions floues, les réseaux dans lesquels ils s'inséraient, l'aspiration à un avenir meilleur qu'ils portaient et étaient censés exaucer, et leurs relations mitigées avec la science, pour n'en citer que quelques-unes ${ }^{2}$.

Bien que les principales organisations de développement soient désormais promptes à dénoncer la tendance à voir les technologies comme des

\footnotetext{
${ }^{1}$ Nous souhaitons remercier tous les contributeurs à ce numéro spécial d'Anthropologie \& développement ainsi que les relecteurs externes et les membres du comité éditorial qui ont participé au processus d'évaluation des différents articles. Dominic Glover, Philippe Lavigne Delville, Janwillem Liebrand et Margreet Zwarteveen nous ont fait part de leurs commentaires sur une version précédente de l'introduction et nous les en remercions.

${ }^{2}$ Les exemples identifiés ici sont issus de nos trajectoires : nous avons tous deux travaillé sur les questions de gestion de l'eau agricole en Asie du Sud et du Sud-Est, en Afrique Subsaharienne, dans la région méditerranéenne et en Asie centrale au cours des dix dernières années. Comme l'illustre ce numéro spécial, d'autres dispositifs existent dans d'autres domaines.
} 
solutions miracles (voir, par exemple, la FAO, 2017, dans le champ de l'agriculture; l'OMS, 2010, concernant le développement d'un vaccin contre le paludisme; la Banque mondiale, 2017, sur la question des technologies de l'information et de la communication dans l'ensei-

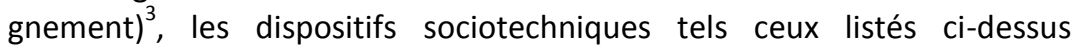
continuent d'être présentés comme des solutions quasi-universelles. On leur prête le pouvoir de résoudre des questions multiples et liées pour in fine fournir $L A$ réponse au développement. À partir des contributions qui suivent, nous allons mettre en lumière les ressemblances, mais aussi les différences, entre dispositifs sociotechniques et technologies dont on avait prédit qu'elles constitueraient les bases du développement.

Dans ce numéro spécial d'Anthropologie \& développement, nous examinons le phénomène des "mythes sociotechniques" dans le développement. II nous faut tout d'abord clarifier la terminologie que nous employons. Le terme de "mythe" nous sert à désigner un type d'intervention qui prétend fournir une solution à des problèmes globaux. Ces mythes sont à la fois clairement délimités et peu précisément définis, si bien que les débats autour de leur consistance empirique en deviennent des éléments constitutifs. II ne s'agit pas pour nous de les rejeter comme "faux » ou " erronés » (une compréhension du terme de mythe à la fois familière et normative), mais d'adopter une approche plus ethnographique afin de comprendre la genèse et le fonctionnement de ces mythes sociotechniques, et ce dans le champ du développement.

Les mythes ne sont pas spécifiques au développement, mais ce qui rend les mythes " dans le développement " différents de leurs homologues dans d'autres domaines est qu'ils se fondent sur (et expriment) un impératif moral à agir pour un avenir meilleur - une aspiration difficile à remettre en question. En outre, la " technostructure » du développement offre un environnement favorable à la construction de mythes. Comme le

${ }^{3}$ Ces affirmations présentent des similarités frappantes: bien qu'elles commencent (et finissent) en soulignant qu'il n'existe aucune solution miracle au problème considéré, elles se limitent à identifier le potentiel d'une technologie spécifique - ou d'une série d'options pour résoudre ce même problème. 
soulignent en effet les tenants d'une analyse des pratiques du développement centrée sur les acteurs :

" une intervention [de développement constitue] une 'réalité multiple' faite de perceptions culturelles et d'intérêts sociaux différents, et caractérisée par des luttes sociales et politiques quotidiennes opposant les acteurs sociaux impliqués " (Long et Van der Ploeg, 1989 : 226).

Cette technostructure est par conséquent propice aux débats et disputes constitutifs des mythes. Les logiques et pratiques d'organisations de développement concurrentes sont peut-être plus importantes encore en ce qui concerne la production de mythes. Comme cela a été démontré depuis longtemps par les anthropologues du développement, ces logiques et pratiques sont largement déconnectées des réalités de terrain (Naudet, 1999, décrit par exemple le développement comme des pratiques visant à « trouver des problèmes à des solutions » toutes faites).

En dépit de la rhétorique sur l'inadéquation des approches de type " remède miracle » (silver bullet en anglais), les interventions standardisées qui promettent une application universelle et des impacts à grande échelle restent attractives pour les acteurs du développement, et leur permettent aussi de légitimer leur existence. D’un autre côté, les administrations nationales ont peu intérêt à ne pas accepter ces interventions qui drainent des financements importants dont elles sont devenues dépendantes. Une autre spécificité du secteur du développement a trait à "l'évaluation » : comme celle-ci ne met que rarement en question la logique des interventions (qui s'ancrent dans un impératif moral à agir ; voir Long et Van der Ploeg, 1989 ; Li, 2007), elle tend à justifier les interventions ultérieures sur la base de promesses futures et joue par conséquent un rôle clé dans la genèse des mythes.

Les articles du numéro spécial abordent tout un éventail de mythes sociotechniques; certains ont une renommée mondiale (comme les vaccins ou les essais contrôlés randomisés), tandis que d'autres sont surtout connus des spécialistes d'un champ donné (comme le système d'intensification rizicole ou les dispositifs d'approvisionnement en eau potable). Les contributeurs s'inspirent d'un large panel de disciplines (anthropologie du développement, écologie politique, études des sciences 
et technologies, géographie humaine) et utilisent la notion de " mythe " sur des modes différents pour éclairer des processus qui dépassent nos champs de travail propres. Certaines contributions nous amènent à faire le lien avec d'autres concepts comme ceux de modèle (par exemple Rap, ce numéro), instrument (Werner, ce numéro), ou mécanisme (Abdelgahfour, ce numéro), ce qui nous oblige à clarifier les relations entre ces concepts (et d'autres) dans la section suivante de cette introduction. Les contributions partagent néanmoins de fortes similarités, confirmant notre hypothèse selon laquelle les processus que nous avons observés dans les pratiques autour de l'irrigation au goutte-à-goutte, de l'agriculture de conservation et du SRI n'étaient pas des coïncidences, mais le signe que les mythes sociotechniques constituent un phénomène bien plus large des pratiques de développement et de la production des politiques publiques.

Dans cette courte introduction structurée en trois sections, nous soulignons certaines des similarités et différences entre les contributions à ce numéro. Premièrement, dans la section "Qu'est-ce qu'un mythe sociotechnique?", nous affinons et dessinons notre approche conceptuelle et proposons une définition de la notion de "mythe sociotechnique " qui s'appuie sur une littérature anthropologique déjà riche et sur d'autres concepts plus largement utilisés. Nous discutons ensuite de l'idée du "Mythe comme performance" et analysons: "Comment les mythes sociotechniques émergent et que font-ils? ". Enfin, nous montrons pourquoi l'étude des mythes sociotechniques est importante, en tenant compte du fait que ces processus ont des implications fortes sur les pratiques et perceptions du développement.

Qu'est-ce qu'un mythe sociotechnique?

Selon la définition de l'Oxford English Dictionary, un mythe est :

" un récit traditionnel, portant en particulier sur l'histoire ancienne d'un peuple ou expliquant un phénomène naturel ou social, et impliquant typiquement des êtres ou événements surnaturels » (Oxford English Dictionary, 2017a).

Cette acception du mythe est largement répandue, et souvent associée à l'adjectif « mythologique ». Une seconde signification du mythe, liée à la 
première - toujours selon l'Oxford English Dictionary (2017a) - est celle d' " une croyance ou idée largement partagée mais fausse ". Ces deux définitions tendent à opposer le " mythe " à la " réalité ", dualité mise en question par les anthropologues qui ont attiré l'attention sur l'imbrication étroite des deux concepts. Selon une lecture anthropologique, les mythes donnent du sens, motivent l'action et, parce qu'ils sont forgés sur un mode allégorique, ils permettent le maintien de l'ordre social tout en ouvrant un espace à sa remise en cause (voir Weiner, 1994, pour une discussion sur le mythe et le langage en anthropologie; voir aussi Sen et al., et Rap, ce numéro, pour une discussion de la notion de mythe).

Dans l'imaginaire commun, le mythe est généralement associé à des termes tels que ceux d'histoire ou de conte (Werner, ce numéro, joue, de fait, sur cette association dans son récit "The Prince and the Magic Magnet »). Selon cette acception du terme, un mythe doit donc être ignoré si l'objectif est de comprendre la "réalité ». S'inspirant d'une vaste littérature sur la performativité du mythe (par exemple Campbell, 1988), ce numéro spécial part d'un point de vue différent, qualifié par certains des contributeurs de compréhension anthropologique du mythe (voir Sen et al., ce numéro). Selon nous, les mythes ne sont pas de simples histoires ; ils sont très réels au sens où ils sont activement forgés et reproduits par des individus - qui peuvent sincèrement y croire, ou pas, pour différentes raisons -, au sein de réseaux qui forment des coalitions sur lesquelles ces mythes s'appuient. À l'instar des discours de Foucault, les mythes contribuent aux manières de voir le monde et d'agir sur lui. Les mythes jouent ce rôle selon des modalités spécifiques, justifiant notre usage du mot. En effet, tout comme dans l'acception commune du terme, les mythes dans le développement mobilisent des croyances et des héros pour changer une situation pour le meilleur, et les promesses futures prennent le pas sur les conditions présentes.

Notre compréhension du " mythe " s'appuie sur d'autres concepts plus largement utilisés dans le champ des études du développement, tels que ceux de " panacée " (Ostrom et al., 2007) ou de "récit " (Roe, 1991). Sen et al. (ce numéro) et Rap (ce numéro) font aussi référence aux récits dans leurs études portant respectivement sur le SRI et la production des politiques d'irrigation, tandis que Liebrand (ce numéro) dépeint les cartes comme des représentations graphiques de récits. Dans son travail sur la 
gouvernance des interactions humains-environnement, Elinor Ostrom emploie le mot "panacée " pour souligner la prévalence et les limites de l'application d' "une solution unique à tous les problèmes " environnementaux (Ostrom et al., 2007). En revanche, elle ne discute pas véritablement le concept d'un point de vue théorique, autrement qu'en indiquant que les remèdes miracles dépassent la portée des solutions techniques. Roe (1991) a montré dans ses travaux comment des récits impulsaient et légitimaient certaines pratiques du développement malgré la remise en cause croissante de leurs mérites empiriques. Il attribue cette persistance à la structure narrative des récits qui partent souvent d'un scénario de crise, pour ensuite fournir un cadre explicatif et proposer une feuille de route (un modèle causal destiné à réduire l'incertitude) visant à résoudre cette crise réelle ou potentielle. Roe (1991) considère que les "récits» sont moins normatifs que l'« idéologie» et plus programmatiques que le "mythe", qu'il associe aux termes de "sagesse populaire ", ou "conte folklorique ". Il souligne que le mot " mythe » est souvent utilisé de façon péjorative, pour écarter des événements, et de ce point de vue, que l'usage du concept peut tendre à obscurcir notre compréhension des processus de développement - point sur lequel nous sommes en désaccord pour les raisons énoncées dans cette introduction.

Nous soutenons que le concept de "mythe " permet d'aller plus loin que ces travaux ayant éclairé les domaines du développement et de la production des politiques publiques. Ce concept est de fait porteur d'un double sens. Tout d'abord, il reconnaît que les " histoires » et " récits " (au sens de Roe, 1991) sont des pourvoyeurs de sens et qu'ils stimulent et légitiment les actions. Plusieurs contributions à ce numéro spécial montrent par exemple comment des interventions de développement particulières acquièrent un caractère mythique via l'élaboration d'un récit convaincant. Sen et al. soulignent ainsi que :

" un mythe fonctionne au travers d'un récit pour rationaliser les perturbations de la vie quotidienne et offrir aux gens des motivations pour ne pas rejeter le changement " (Sen et al., ce numéro).

C'est une question sur laquelle nous reviendrons dans la section suivante, lorsque nous explorerons "Ce que font les mythes ». Ensuite, le concept permet de souligner que les acteurs du développement sont aussi 
des êtres humains porteurs de cultures, de croyances, d'aspirations et de rituels - y compris les rituels relatifs à la production de "preuves» (Liebrand, ce numéro, offre une analyse de l'élaboration et de l'usage des cartes comme preuves). Le concept de mythe nous rappelle que la " preuve " acquiert pertinence et autorité uniquement au travers de son élaboration et de sa validation au sein de réseaux dans lesquels une interprétation spécifique d'une situation donnée se stabilise (voir Mosse, 2004, sur l'interface entre preuve et interprétation).

Le mot " mythe " est également différent de celui de " modèle " qui a été particulièrement utilisé pour analyser comment des approches standardisées spécifiques "voyagent" d'un lieu à un autre (voir, entre autres, Behrends et al., 2014, et Olivier de Sardan et al., 2017). En effet, le terme de modèle véhicule une image de causalité et de rationalité, qui pourrait conduire les chercheurs à sous-estimer les questions d'agency, de pratiques, d'éthique et d'aspirations qui sont centrales dans le concept de mythe et, selon nous, dans les processus de développement ${ }^{4}$. Le concept de "modèle » a également été utilisé essentiellement en relation avec ceux d' " intervention " et de "politique publique " au risque de négliger les enjeux de matérialité (une exception étant Rottenburg, 2007). Cela est particulièrement problématique dans la mesure où la croyance dans la technologie comme moteur du progrès et du changement constitue une pierre angulaire de la modernité et des pratiques de développement, ce qui signifie que les discours au sujet des technologies (l'interprétation) sont souvent plus performatifs que les technologies elles-mêmes et leur mise en œuvre (la preuve). En outre, nous observons que les récits du développement tournent encore largement autour de la capacité de dispositifs sociotechniques spécifiques (à savoir des assemblages complexes d'objets techniques, de pratiques recommandées et de formes

\footnotetext{
${ }^{4}$ Notons que les auteurs que nous citons, ainsi que d'autres chercheurs en anthropologie du développement comme David Mosse ou Thomas Bierschenk, mettent clairement en évidence que l'élaboration et la diffusion de " modèles " sont enchâssées dans des pratiques et luttes sociales. Nous considérons cependant que ces aspects risquent d'être négligés en raison de l'usage largement répandu du terme " modèle » dans les sciences naturelles (comme cadre explicatif analytique pour représenter les processus naturels). Voir Olivier de Sardan et al. (2017) pour une discussion récente des concepts de modèle, dispositif et mécanisme.
} 
d'organisations permettant leur fonctionnement harmonieux) à résoudre les grands défis auxquels la planète ferait face. Gangneron (ce numéro), par exemple, illustre cela pour les systèmes d'approvisionnement semiurbain en eau potable et Glover et al. (2017) pour l'irrigation au goutte-àgoutte et le système de riziculture intensive.

Tournons-nous à présent vers le second mot clé de ce numéro spécial : le caractère sociotechnique des mythes discutés dans les différentes contributions.

Les technologies ont longtemps joué un rôle central dans les pratiques et les discours du développement. Vues comme les applications directes d'une "science " neutre et objective, les technologies sont devenues dominantes dans les programmes publics d'aide au développement des années 1950 avec une floraison de programmes de transfert technologique. Ces derniers se fondaient sur l'hypothèse de l'universalité de l'applicabilité des objets techniques, indépendamment des contextes socioenvironnementaux de leur usage.

Les technologies furent ainsi promues au rang de "solutions miracles $»^{5}$ qui allaient résoudre les grands problèmes sociétaux tels que la faim, la pauvreté, la maladie ou la dégradation de l'environnement. À partir des années 1970, ces programmes durent affronter des critiques de plus en plus nombreuses, à mesure qu'il apparaissait qu'ils ne réduisaient pas la pauvreté et qu'ils pouvaient même engendrer un accroissement des inégalités. Dans le secteur agricole, par exemple, les technologies constitutives de la "Révolution verte " commencèrent à être violemment remises en question - et elles le sont toujours. Les critiques se concentraient en particulier sur le rôle central donné au savoir basé sur la science et l'ingénierie (bien souvent au détriment d'autres formes de savoirs; voir entre autre Glover et al., 2017) et sur le fait que les programmes de transfert technologique ignoraient largement la nature systémique des processus d'innovation (voir par exemple Geels, 2004,

\footnotetext{
${ }^{5}$ Selon la légende, seule une balle en argent peut tuer le loup-garou mythique. Selon l'Oxford English Dictionary, "silver bullet " (traduit en français par "solution miracle ») signifie " une solution simple et apparemment magique à un problème compliqué " (Oxford English Dictionary, 2017b).
} 
pour un argumentaire général, ainsi que Biggs, 1990, et les chapitres réunis dans Chauveau et al., 1999, et Coudel et al., 2013, pour une application au secteur agricole).

Ainsi, dans les années 1980 et 1990, le mythe du "développement par la technologie " sembla se dissiper. Était-ce bien le cas ? Nul doute, les agences de développement sont maintenant promptes à souligner qu'il n'existe pas de "technologie miracle " face aux défis du développement, ou encore qu'il n'existe aucune " approche modèle " (blueprint approach). Le changement terminologique - de " miracle " à " modèle " - n'est pas neutre et montre que les organismes de développement ont pour partie internalisé le besoin de dépasser le "tout technologique ". Mais le vocabulaire utilisé provient toujours de l'ingénierie (après tout, une " impression en bleu » (blueprint), est une reproduction d'un plan ou d'un dessin technique caractérisée par des lignes légèrement teintées sur un fond bleu, procédé largement employé dans le champ de l'architecture et de l'industrie entre les années 1860 et 1940). Le mythe de l'intervention planifiée analysé par Long et Van der Ploeg (1989) continue d'irriguer les cultures, logiques et pratiques des individus et des organisations impliqués dans le secteur du développement, comme le soulignent aussi les contributions de Liebrand, ce numéro, et Rap, ce numéro (voir aussi Scott, 1998 ; Lavigne Delville, 2012). Mosse (2004) nous rappelle qu'il s'agit de stratégie et pas d'aveuglement: plans et modèles servent à aligner des intérêts, forger des alliances, et mobiliser des fonds et des appuis. Ils ont un rôle pivot dans la construction des réseaux sociotechniques au sein desquels se déploient les dispositifs sociotechniques.

L'époque à laquelle les " technologies modernes " constituent le point de départ du développement est loin d'être terminée. Plusieurs contributions à ce numéro spécial nous rappellent clairement à quel point les objets technologiques et la science vue comme source objective de savoir, continuent de fasciner de nombreux acteurs et restent centraux dans la pratique du développement. Le premier aspect est exemplifié par les contributions de Werner (sur l'imagerie par résonance magnétique IRM) et Thiongane et al. (sur les vaccins); le second par la contribution d'Abdelghafour sur l'approche de l'évaluation par essais contrôlés randomisés (ECR). Néanmoins, dans de nombreux cas, la technologie n'arrive pas seule, mais est promue comme partie d'un dispositif 
sociotechnique plus large incluant un ou plusieurs artefacts mais aussi un mode opératoire (un mécanisme clarifiant comment l'artefact doit être utilisé et par qui), et un dispositif organisationnel dans lequel il doit s'insérer pour un "usage efficace ${ }^{6}$. Selon nous, ces dispositifs sociotechniques sont même plus puissants que les technologies antérieures, et ce parce qu'ils sont représentés, évalués, discutés et mis en œuvre différemment selon les acteurs - tout en conservant une certaine cohérence. À l'instar des objets frontières (Star et Griesemer, 1989), ils sont nettement délimités pour se conformer à une prétention à l'universalité tout en offrant une marge de manœuvre à l'interprétation et l'ajustement: les technologies étaient (relativement) statiques, les dispositifs sociotechniques sont dynamiques.

Si certaines technologies spécifiques pouvaient être assez facilement rejetées parce qu'elles étaient inadaptées à leur contexte de mise en œuvre (l'écart entre le potentiel présumé d'un artefact donné et sa nonadoption a pour partie motivé la critique du modèle diffusionniste de Rogers, 1983), c'est plus difficilement le cas pour les dispositifs sociotechniques. Ceux-ci se réfèrent explicitement à l'importance du " contexte » et l'une des raisons de leur attrait est qu'ils manifestent une malléabilité ${ }^{7}$ suffisante pour être ajustés à celui-ci. Le système de riziculture intensive (Sen et al., ce numéro, et Serpantié, ce numéro), l'irrigation au goutte-à-goutte (Venot, 2016) et l'agriculture de conservation (Giller et al., 2009) constituent des illustrations claires de ce

\footnotetext{
${ }^{6}$ Philippe Lavigne Delville remarque que les dispositifs dont nous parlons sont «technicoinstitutionnels » ou " technico-organisationnels " plutôt que sociotechniques dans la mesure où ils font largement l'impasse sur les dynamiques sociales et se limitent souvent à des recommandations standardisées concernant le dispositif organisationnel ou institutionnel au sein duquel un procédé spécifique doit être employé. Nous préférons retenir l'expression "dispositif sociotechnique " - c'est une manière de souligner que les dispositifs que nous discutons sont enchâssés dans des réseaux sociotechniques et édictés par ces derniers.

7 De Laet et Mol (2000) par exemple attribuent le "succès" de la "pompe de brousse " zimbabwéenne à sa malléabilité (ils utilisent le concept de fluidité qui va au-delà de la seule capacité technique à s'ajuster au contexte de mise en œuvre).
} 
fait ${ }^{8}$. En outre, certains mythes sociotechniques ont moins à voir avec les objets technologiques qu'avec "la manière dont ils atterrissent dans de nouveaux contextes »: voir par exemple les entreprises sociales pour I'irrigation au goutte-à-goutte (Venot, 2016) et les partenariats public-privé pour le MenAfricVac ${ }^{\circledR}$ (Thiongane et al., ce numéro). Enfin, les débats (scientifiques et entre praticiens) portant sur les "frontières" de tout dispositif sociotechnique (de quoi est-il fait), son domaine d'applicabilité et ses impacts font désormais partie intégrante du dispositif lui-même, et ne sont plus extérieurs, comme le montrent clairement les cas des essais contrôlés randomisés (Abdelghafour, ce numéro), du MenAfriVac ${ }^{\circledR}$ (Thiongane et al., ce numéro) et du SRI (Serpantié, ce numéro).

Dans la section qui suit, nous décrivons les processus qui sous-tendent la formation des mythes sociotechniques et ce qu'ils font (c'est-à-dire leurs performances). Nous montrons qu'ils partagent des points communs avec les propositions techniques antérieures - largement parce qu'ils s'inscrivent dans une compréhension moderniste et linéaire du développement qu'ils contribuent à façonner.

Les mythes comme performance : comment les mythes naissentils et que font-ils?

Nous allons à présent explorer ce que font les mythes sociotechniques dans le développement, comment ils se construisent, se consolident et deviennent opérationnels. Nous soutenons que les mythes sociotechniques dans le développement : 1) créent du sens et motivent l'action, et 2) permettent de générer et de préserver crédibilité et légitimité au sein de réseaux épistémiques.

\footnotetext{
${ }^{8}$ Aujourd'hui, les acteurs du développement ont partiellement intériorisé le fait que les dispositifs sociotechniques doivent prendre en compte le contexte et s'y ajuster, même s'il y a toujours une tendance à l'accuser - et avec lui, les gens qui le peuplent - plutôt qu'à remettre en cause (le potentiel de) l'intervention lorsque les résultats (en termes d'adoption par exemple) ne sont pas à la hauteur des attentes.
} 


\section{Création du sens et " technicisation »}

L'une des caractéristiques communes aux mythes dans le développement est qu'ils appellent à une morale, une aspiration et/ou un espoir supérieurs. Les mythes expriment une vision idéale du monde tel qu'il devrait être, en ce qu'ils aident à imaginer et à représenter quelque chose de non (encore) advenu, mettant l'accent sur le " potentiel » et projetant un futur désirable. Plusieurs contributions abordent ce point : Werner (ce numéro) fournit par exemple un aperçu du rôle des images (au sens propre) dans le cas de l'IRM tandis que Liebrand (ce numéro) analyse la performativité des cartes.

Des connexions et associations sont établies avec une idée abstraite de ce qui est "bon ou bien", ou avec des valeurs supposées universelles (bien qu'elles reflètent une vision étroite du progrès, essentiellement occidentale et masculine), telles que l'équité, le progrès, le développement et la modernité. Comme le soulignent plusieurs auteurs ${ }^{9}$, ces promesses ont une fonction performative et sont des ressources cruciales pour la création de réseaux sociotechniques dans lesquels vont s'insérer des dispositifs spécifiques -, et ce parce qu'elles permettent de détourner l'attention de dynamiques actuelles qui peuvent être contestées.

II ne s'agit pas uniquement d'associations avec des "valeurs" et des "promesses" mais aussi avec les cadres globaux les incarnant afin d'enrôler les acteurs du développement dans des réseaux sociotechniques d'appui. Sans surprise, plusieurs des mythes discutés dans ce numéro spécial sont étroitement liés aux anciens Objectifs du millénaire pour le développement et aux actuels Objectifs du développement durable. Un lien est ainsi établi entre le système de riziculture intensive (SRI) et I'intensification durable et la sécurité alimentaire (Sen et al., ce numéro ; Serpantié, ce numéro) comme cela peut être le cas pour l'irrigation au goutte-à-goutte (Venot, 2016), tandis que le MenAfriVac ${ }^{\circledR}$ (Thiongane et al., ce numéro) et l'IRM (Werner, ce numéro) sont des outils d'amélioration des soins et de progrès en matière de santé et de bien-être. Un

\footnotetext{
${ }^{9}$ Voir par exemple Burkhardt (2001) sur les biotechnologies agricoles et Geels et Smit (2000) sur les technologies de l'information et de la communication (TIC).
} 
système d'approvisionnement en eau potable (Gangneron, ce numéro) est destiné à traiter les besoins en eau potable (et en assainissement). Enfin, les mythes du transfert de la gestion de l'irrigation (Rap, ce numéro) et des essais contrôlés randomisés (Abdelghafour, ce numéro) sont d'une autre nature; ils se rapportent à l'objectif néolibéral de l'efficacité des institutions et de l'usage d'actifs et de ressources (rares).

Des connexions peuvent être faites entre des dispositifs sociotechniques spécifiques et des objectifs plus larges sous la forme de cartes (voir par exemple Liebrand, ce numéro, pour le cas du développement de l'irrigation au Népal), d'images (voir les illustrations dans la contribution de Serpantié, ce numéro). Mais ces connexions passent essentiellement par des récits, c'est-à-dire des histoires comportant un début, un milieu et une fin, et fournissant un cadre explicatif et programmatique (Roe, 1991) sur la manière dont un dispositif sociotechnique va contribuer à réaliser un " bien supérieur ». Les cadres programmatiques suivent en général une structure commune (définition du problème, identification d'une solution potentielle, mise en œuvre et évaluation) et ils affichent un certain degré de nouveauté - ce qui différencie le mythe sociotechnique des approches antérieures. La " nouveauté » de l'IRM (" high-tech »), des ECR (un cadre d'évaluation "solide et objectif ") ou du MenAfriVac ${ }^{\circledR}$ (" abordable ") est claire dans les contributions de Werner (ce numéro), Abdelghafour (ce numéro) et Thiongane et al. (ce numéro). Gangneron (ce numéro) montre aussi la manière dont les systèmes d'adduction d'eau dans les zones semiurbaines sont présentés comme porteurs d'une évolution importante par rapport à d'autres techniques comme les puits creusés à la main et les pompes à motricité humaine. Autre cas typique, la manière dont les promoteurs du SRI ont soigneusement évité le terme " technologie " pour relayer l'idée que ce "kit " constituait d'une manière ou d'une autre une alternative aux approches antérieures d'intensification (Sen et al., ce numéro). Mais la nouveauté ne suffit pas pour qu'un assemblage sociotechnique se transforme en mythe, lequel, comme nous l'avons vu, est vecteur d'aspirations, de valeurs et d'idées (voir par exemple l'analyse de Liebrand, ce numéro, sur le développement planifié de l'irrigation comme véhicule de la « masculinité »).

À ce stade, nous devons nous tourner vers un processus-clé du développement, le processus de " construction de boîtes noires » (" black- 
boxing ") et de "technicisation » (" rendering technical »), pour paraphraser Li (2007) (à savoir la réduction des processus du développement à de simples relations de cause à effet - voir aussi Long et Van der Ploeg, 1989, pour une critique). Plusieurs contributeurs à ce numéro soulignent en effet que l'élaboration des mythes met en action un jeu d'ombres et de lumières qui met sur le devant de la scène certains enjeux, tout en laissant à l'arrière-plan d'autres questions, au risque de marginaliser certains acteurs et points de vue. Cela ressort clairement de l'analyse du MenAfriVac ${ }^{\circledR}$ par Thiongane et al. (ce numéro), où un vaccin - développé contre une souche spécifique de méningite - est paré d'une promesse d'éradication des épidémies de méningite relevant pourtant de sérotypes différents. De même, la contribution de Gangneron (ce numéro) montre qu'au-delà de l'accès universel annoncé, les systèmes d'approvisionnement en eau potable dans les zones semi-urbaines tendent à exclure les plus pauvres qui ne peuvent payer un prix de l'eau plus élevé. Deux processus paraissent centraux dans la "technicisation ". Premièrement, l'identification d'un "succès initial " qui justifie de chercher à le répliquer (voir par exemple Rap, ce numéro). Ensuite, la standardisation - la recherche de ressemblances et d'abstraction pour partie motivée par une "volonté d'amélioration" ( $L i, 2007)$, et qui se fait via la production de " recommandations " et d'activités de " conditionnement " (Glover et al., 2017) qui permettent au dispositif sociotechnique de voyager - précondition à l'élaboration du mythe.

\section{Motiver l'action au sein de coalitions de soutien}

Les processus décrits dans la section précédente façonnent et se déploient dans des réseaux sociotechniques étendus, mais qui partagent un trait commun: ils sont centrés autour d'un ou de plusieurs "héros" qui figurent en bonne place dans le récit qui sous-tend le mythe et portent le changement grâce à leur personnalité remarquable (Liebrand, ce numéro, nous rappelle que ce sont souvent des hommes, et avance un argument sur le lien entre les idées occidentales de progrès et la masculinité).

La personnification est en effet un autre élément des mythes sociotechniques souligné par différents contributeurs (Thiongane et al., ce numéro, attirent par exemple notre attention sur Marc LaForce ; Serpantié, ce numéro, sur Fr. Henri de Laulanié et Norman Uphoff; Liebrand, ce 
numéro, sur l'« Homme de l'irrigation » et l'« Empereur de l'eau " au Népal). Dans la théorie de l'acteur réseau, ces héros sont connus comme "Le Prince » (Werner, ce numéro), le " porte-parole " ou l'" entrepreneur (schumpétérien) " (Akrich et al., 1988a). Ils jouent un rôle clé dans la " création de l'intéressement " (Akrich et al., 1988b) et l'établissement d'un réseau d'acteurs dense mais multiforme (une coalition de soutien) au travers duquel le mythe accroît sa portée (voir par exemple Abdelghafour, ce numéro, et Thiongane, ce numéro, qui décrivent respectivement les coalitions autour des essais contrôlés randomisés et le MenAfriVac $\left.{ }^{\circledR}\right)$. Rap (ce numéro) montre aussi que ces entrepreneurs ne sont pas des figures immuables et que certains individus (dans son cas des agents du ministère en charge de l'irrigation au Mexique) peuvent émerger comme des porteparole particulièrement puissants au cours de l'élaboration du mythe, parce qu'ils agissent et représentent des collectifs spécifiques au sein du réseau sociotechnique.

Dans ce type de réseaux, le mythe inspire, convainc, mobilise et agrège, ce qui conduit les personnes qui sont extérieures à qualifier leurs membres d' "adeptes " ou de "croyants ", dans un parallèle avec les mouvements religieux. Giller et al. (2009) vont clairement dans ce sens en intitulant de manière provocatrice leur article sur l'agriculture de conservation « an heretic's view ». Plusieurs des contributeurs à ce numéro spécial (Liebrand, Rap, Sen et al., Thiongane) font aussi le lien avec la religion lorsqu'ils mettent l'accent sur les « rituels " qu'implique la création d'un mythe.

Les croyances, la volonté de progrès et les aspirations à un bien supérieur ne sont pas toutefois le seul moteur de l'élaboration des mythes, loin de là. Cette élaboration est menée de façon stratégique par divers acteurs parce que le mythe est en accord avec leurs intérêts, leur permet de faire avancer leurs agendas et, en retour, rend leurs actions légitimes. Le réseau tire sa force de deux caractéristiques: 1) un fort niveau d'ingéniosité et d'adaptabilité dans la redéfinition du dispositif sociotechnique (ou plutôt dans le choix de mettre en lumière certains aspects et d'en laisser d'autres dans l'ombre), et 2) une capacité à s'ordonner et à s'unir derrière une morale commune face aux critiques externes. De fait, le réseau offre un espace de stabilisation du mythe via l'élaboration stratégique d'une interprétation particulière des événements et la 
construction de la notion de succès (voir par exemple Rap, ce numéro, Thiongane, ce numéro, et Mosse, 2005, pour un argument générique).

\section{Création et préservation de la crédibilité : une relation dialectique avec la science}

Si les mythes mobilisent et renforcent leurs réseaux de soutien en faisant référence à un objectif louable, ils interviennent aussi dans les dynamiques de production des savoirs et de leur autorité. Cela nécessite un équilibre entre, d'une part, une histoire simple de cause à effet, facile à saisir et à vendre, et, d'autre part, une accroche plus systématique du récit.

II existe une relation dialectique entre mythe et science ; la science est utilisée afin de rendre le mythe légitime (enrôlement d'universités et de chercheurs, publications), mais elle peut également être écartée en tant que savoir " expert », déconnecté des réalités du terrain et de l'action.

Les articles de ce numéro illustrent cette dualité. Le mythe de l'ECR (Abdelghafour, ce numéro) s'ancre dans une vision normative de la science (plus spécifiquement dans la supériorité de " statistiques non biaisées ") et de la relation entre science et politique, qui est particulièrement attractive pour les agences de développement et les fondations privées. En tant que " mythe d'évaluation ", le mythe de l'ECR est particulièrement puissant pour ce qui est de rendre légitime certaines idéologies (dans ce cas, la nécessaire efficacité économique de l'utilisation des ressources financières), mais fortement critiqué par la communauté des chercheurs en sciences sociales. À l'opposé, le mythe SRI se structure autour de la notion d' "observations de terrain " à l'opposé des principes directeurs d'une science rizicole "dominante" qui avait sous-tendu la Révolution verte dans les années 1960 et 1970, au point que Serpantié (ce numéro) en appelle à une "recherche agronomique sérieuse " capable de mieux apprécier la dynamique et les impacts du SRI.

La question des indicateurs permettant d'évaluer la validité et la légitimité d'une intervention est souvent centrale dans les débats scientifiques autour des mythes sociotechniques. On observe dans de nombreux cas des (auto-)évaluations soigneusement orchestrées (voir Abdelghafour, ce numéro, sur les ECR ; Serpantié, ce numéro, sur le SRI ; et Venot, 2016, sur l'irrigation au goutte-à-goutte). La conduite d'analyses en 
interne permet de sélectionner les statistiques favorables (nombres de kits de goutte-à-goutte vendus, nombre de paysans ayant adopté un package spécifique, nombre d'individus vaccinés, nombre de branchements au réseau d'adduction d'eau, etc.) qui peuvent être présentées au " monde extérieur " tandis que d'autres aspects peuvent être retravaillés en interne comme points à améliorer. Ici encore, les promesses futures prennent le pas sur les observations présentes.

De plus, dans la mesure où les mythes sociotechniques proposent des définitions souvent ouvertes/lâches à la fois des dispositifs et des contextes, ils sont à même de se défendre et de discréditer les études critiques en les renvoyant à une mise en œuvre partielle du package et/ou un contexte d'application défavorable. La malléabilité du mythe permet tout autant de fonder une prétention à l'universalité que de se décharger de toute responsabilité si les choses ne se passent pas comme prévu.

Notre argument ici est que conduire de nouvelles recherches - qu'elles qu'en soient les formes - n'aura probablement aucune influence sur les dynamiques d'élaboration des mythes. Au contraire, les controverses (scientifiques) font partie intégrante du processus comme le montrent aussi Sen et al. (ce numéro). Les mythes sociotechniques sont, de fait, caractérisés par des affirmations, voire des exagérations divergentes qui prêtent à des discussions enflammées et à des débats polarisés. Se référant à Gregory Bateson, Stone (2015) dans son étude sur les biotechnologies nommait "schismogenèse " ces phénomènes autoamplificateurs de divergence. Ces processus ne sont pas fortuits; les débats scientifiques - parfois vigoureux - au sujet de l'agriculture de conservation (Giller et al., 2009), l'irrigation au goutte-à-goutte (Kuper et al., 2017) ou les vaccins contre la méningite (Thiongane et al., ce numéro), pour n'en citer que quelques-uns, contribuent à "faire connaître " ces dispositifs sociotechniques. II se pourrait bien que les réseaux socio-techniques auxquels s'adossent les mythes sociotechniques soient forgés de manière à créer les conditions de tels débats, une façon de faciliter le désaccord organisé et d'attirer l'attention. Après tout, si les chercheurs discutent ces mythes - positivement ou négativement -, c'est en soi un signe de leur intérêt. Cela pose la question de savoir comment des chercheurs critiques en sciences sociales peuvent aborder ces mythes de façon constructive. 


\section{Pourquoi et comment traiter des mythes sociotechniques?}

Selon nous, le concept de mythe sociotechnique permet d'éclairer les pratiques et politiques de développement et les raisons expliquant pourquoi certaines de ces interventions accèdent au statut de "modèle appelant la réplication ". Cela tient à l'élaboration d'une interprétation stabilisée mais malléable de la nature et des promesses de ces interventions, une interprétation qui est stratégique et minutieusement élaborée au sein de coalitions de soutien à la fois spécifiques et étendues. Le concept invite aussi à ouvrir certaines des "boîtes noires" qui caractérisent les pratiques de développement, permettant ainsi de mettre au jour les motivations et idéologies qui les sous-tendent et les risques de marginalisation qui peuvent exister.

En tant que chercheurs en sciences sociales, notre relation aux mythes sociotechniques est duale. II y a tout d'abord une inclination claire vers la critique, très visible dans plusieurs articles de ce numéro. Les auteurs justifient ce positionnement par le fait que les mythes sociotechniques simplifient une réalité complexe, légitiment des réformes en accord avec des idéologies dominantes profondément inégalitaires, et génèrent par conséquent de la marginalisation. Quelle que soit la pertinence de ces critiques (et nous partageons de fait l'essentiel de ces points de vue), elles seront peu à même de changer l'état des choses. Au contraire, elles risquent de renforcer les mythes qu'elles veulent discréditer (voir aussi Liebrand, ce numéro, pour un argument similaire). Dans la mesure où les débats scientifiques (et parfois houleux) sont des éléments-clés de la production des mythes sociotechniques, une approche scientifique positiviste sera incapable de les endiguer.

Cela ne signifie pas qu'il faille nous dérober face à la complexité des pratiques du développement, et notamment ne pas nous engager dans des activités de construction de coalitions en faveur de ce qui nous paraît important et juste. Certains pourraient dire que ce n'est pas le rôle des chercheurs qui doivent garder une certaine distance face aux événements - afin de pouvoir les comprendre. Mais rester dans sa tour d'ivoire est aussi un choix politique - un choix fort qui contribue également à forger les réalités du développement, mais en des termes décidés par d'autres. Les mythes ont une capacité à libérer un incroyable niveau d'énergie et de 
créativité parmi une grande diversité d'acteurs et cette qualité pourrait de fait être mise à contribution ; comme Sen et al. (ce numéro) l'énoncent, les mythes, en raison de leur malléabilité, " peuvent [aussi] guider les gens et leurs communautés dans la gestion du changement sans prédéterminer de solutions toutes faites ". L'enjeu est donc de discuter de manière constructive les mythes et les cadres normatifs dominants qui les sous-tendent et, pour les plus engagés d'entre nous, de forger des mythes alternatifs aux orientations différentes.

\section{Bibliographie}

AKRICH M., CALLON M., LATOUR B., 1988a, "À quoi tient le succès des innovations? 2: le choix des porte-parole ", Gérer et comprendre, Annales des Mines, 12 : 14-29.

AKRICH M., CALLON M., LATOUR B., 1988b, "À quoi tient le succès des innovations ? 1 : L'art de l'intéressement ", Gérer et comprendre, Annales des Mines, $11:$ 4-17.

BANQUE MONDIALE, 2017, "Education provides the analogue foundation for our digital lives ", by Michael Trucano, http://blogs.worldbank.org/ edutech/education-provides-analogue-foundation-our-digital-lives, Last visited 31/08/2017.

BEHRENDS A., PARK S.J., ROTTENBURG R., 2014, " Travelling Models: Introducing an Analytical Concept to Globalisation Studies ", in BEHRENDS A., PARK S.J., ROTTENBURG R. (eds.), Travelling Models in African Conflict Management. Translating Technologies of Social Ordering, Leyden, Brill: 1-40.

BURKHARDT J., 2001, "Agricultural biotechnology and the future benefits argument ", Journal of Agricultural and Environmental Ethics, 14 : 135145 .

BIGGS S.D., 1990., " A multiple Source of innovation model of agricultural research and technology promotion ", World Development, 38(1) : 1481-1499.

CAMPBELL J., 1988, The power of myth, New York, Doubleday. 
CHAUVEAU J.P., CORMIER SALEM M.C., MOLLARD E. (éds), 1999, L'innovation en agriculture : questions de méthodes et terrains d'observation, Paris : IRD, $362 \mathrm{p}$.

COUDEL E., DEVAUTOUR H., FAURE G., SOULARD C. et HUBERT B., 2013, "Renewing innovation systems in agriculture and food. How to go towards more sustainability?", Wageningen, The Netherlands, Wageningen Academic Publishers.

DE LAET M. et MOL A., 2000, "The Zimbabwe Bush Pump: Mechanics of a fluid technology ", Social Studies of Science, 30(2) : 225-263.

FOOD AND AGRICULTURAL ORGANIZATION OF THE UNITED NATIONS (FAO), 2017, "Reduce rural poverty », http://www.fao.org/about/what-we-do/so3 /en/, Last visited 31/08/2017.

GEELS F.W., 2004, «From sectoral systems of innovation to socio-technical systems: Insights about dynamics and change from sociology and institutional theory ", Research policy, 33 : 897-920.

GEELS F.W., SMIT W.A., 2000, "Failed technologies futures: pitfalls and lessons from a historical perspective ", Futures, $32: 867-885$.

GILLER K.E., WITTER E., CORBEELS M. et TITTONELL P., 2009, «Conservation agriculture and smallholder farming in Africa: The heretics' view ", Field Crops Research, 114(2009) : 23-34.

GLOVER D, VENOT J.P. et MAAT H., 2017, « On the movement of agricultural technologies: packaging unpacking and situated reconfiguration ", in SUMBERG T. (éd.), Agronomy for Development: The politics of knowledge in agricultural research, London and New York, Routledge : 14-30.

LAVIGNE DELVILLE P., 2012, «Affronter l'incertitude? Les projets de développement à contre-courant de la 'révolution du management de projet' ", Revue Tiers Monde, 211 : 153-168.

KUPER M., VENOT J.P. et ZWARTEVEEN M., 2017, « Introduction: Panda or Hydra? The untold stories of drip irrigation ", in VENOT J.P., KUPER M. et ZWARTEVEEN M. (éds), Drip irrigation for Agriculture: untold stories of efficiency, innovation and development, Routledge, Abingdon \& New York : 1-15.

LI T.M., 2007, The will to improve. Governmentality, development, and the practice of politics, Durham, Duke University Press. 
LONG N. et VAN DER PLOEG J.D., 1989, « Demythologizing planned intervention: An actor perspective ", Sociologia Ruralis, 29(3/4) : 226-249.

MOSSE D., 2004, "Is good policy unimplementable? Reflections on the ethnography of aid policy and practice ", Development and Change, 35(4) : 639-671.

MOSSE D., 2005, Cultivating development. An ethnography of aid policy and practice, London, Pluto Press.

NAUDET J.D., 1999, Trouver des problèmes aux solutions : 20 ans d'aide au Sahel. Paris, OCDE.

OLIVIER DE SARDAN J.P., DIARRA A. et MOHA M., 2017, "Traveling models and the challenges of pragmatic contexts and practical norms; the case of maternal health ", Health Research Policy and Systems, 15(Suppl. 1) : 7187.

ORGANISATION MONDIALE DE LA SANTÉ (OMS), 2010, «A research agenda for malaria eradication ", by Dr Margaret Chan Director-General of the WHO, http://www.who.int/dg/speeches/2010/malaria_20100326 /en/, Last visited 31/08/2017.

OSTROM E., JANSSEN M.A. et ANDERIES J.M., 2007, "Going beyond panaceas ", Proceedings of the National Academy of Sciences, 104(39) : 15176-15178.

OXFORD ENGLISH DICTIONARY, 2017a, " Myth », https://en.oxforddictionaries.com /definition/myth, Last visited 31/08/2017.

OXFORD ENGLISH DICTIONARY, 2017b, "Silver bullet », https://en.oxford dictionaries.com/definition/silver_bullet, Last visited 31/08/2017.

ROE E.M., 1991, "Development narratives, or making the best of blueprint development », World Development, 19(4) : 287-300.

ROTTENBURG R., 2007, Far-fetched facts. A parable of development aid, Cambridge, MIT Press.

ROGERS E.M., 1983, Diffusion of Innovations, $3^{\mathrm{e}}$ édition, New York, MacMillan.

SCOTT J.C., 1998, Seeing like a state - How certain schemes to improve Human condition have failed, New Haven and London, Yale University Press.

STAR S.L. et GRIESEMER J.R., 1989, «Institutional ecology. 'Translations' and boundary objects: Amateurs and professionals in Berkeley's museum of vertebrate zoology ", Social Studies of Sciences, 19(3) : 387-420. 
STONE G.D., 2015, "Biotechnology, Schismogenesis, and the demise of Uncertainty ", Journal of Low and Policy, 47(1) : 29-49.

VENOT J.P., 2016, "A success of some sort: drip irrigation, social enterprises and drip irrigation in the developing world ", World Development, 79(3) : 6981.

WEINER J.F., 1994, "Myth and metaphor », in INGOLD T. (éd.), Companion Encyclopedia of Anthropology, London and New York, Routledge : 591612.

\author{
Niviviv \\ Jean-Philippe Venot est chargé de recherche en géographie et études du développement \\ IRD, UMR G-EAU, université de Montpellier (France) \\ et Water resources Management Group, Wageningen University (Pays-Bas) \\ E-mail : jean-philippe.venot@ird.fr
}

Gert Jan Veldwisch est assistant professeur, Eau \& Développement

Water Resources Management Group, Wageningen University (Pays-Bas)

E-mail : gertjan.veldwisch@wur.nl 